\title{
Sinc collocation linked with finite differences for Korteweg-de Vries Fractional Equation
}

\author{
Kamel Al-Khaled \\ Department of Mathematics and Statistics, Jordan University of Science and Technology, Jordan
}

\section{Article Info}

Article history:

Received Apr 9, 2019

Revised Jul 28, 2019

Accepted Aug 30, 2019

\section{Keywords:}

Fractional derivative Korteweg-de Vries Equation

ABSTRACT

A novel numerical method is proposed for Korteweg-de Vries Fractional Equation. The fractional derivatives are described based on the Caputo sense. We construct the solution using different approach, that is based on using collocation techniques. The method combining a finite difference approach in the time-fractional direction, and the Sinc-Collocation in the space direction, where the derivatives are replaced by the necessary matrices, and a system of algebraic equations is obtained to approximate solution of the problem. The numerical results are shown to demonstrate the efficiency of the newly proposed method. Easy and economical implementation is the strength of this method.
\end{abstract} Numerical solutions

Sinc-Collocation

\section{Corresponding Author:}

Kamel Al-Khaled, Jordan University of Science and Technology, Irbid, P.O.Box 3030, Jordan.

Email: kamel@just.edu.jo
Copyright (C) 2020 Insitute of Advanced Engineeering and Science. All rights reserved.

\section{INTRODUCTION}

Nonlinear partial differential equations appear in many branches of physics, engineering and applied mathematics. In recent years, there has been a growing interest in the field of fractional calculus. Oldham and Spanier [1], Miller and Ross [2], and Podlubny [3] provide the history and a comprehensive treatment of this subject. Fractional calculus is the field of mathematical analysis, which deals with the investigation and applications of integrals and derivatives of arbitrary order, which can be real or complex. The subject of fractional calculus has gained importance during the past three decades due mainly to its demonstrated applications in different areas of physics and engineering. Several fields of applications of fractional differentiation and fractional integration are already well established, some others just started. Many applications of fractional calculus can be found in turbulence and fluid dynamics, stochastic dynamical systems, plasma physics and controlled thermonuclear fusion, nonlinear control theory, image processing, nonlinear biological systems. It is important to solve time fractional partial differential equations. It was found that fractional time derivatives arise generally as infinitesimal generators of the time evolution when taking along time scaling limit. Hence, the importance of investigating fractional equations arises from the necessity to sharpen the concepts of equilibrium, stability states, and time evolution in the long time limit. There has been some attempt to solve linear problems with multiple fractional derivatives. In [4], an approximate solution based on the decomposition method is given for the generalized fractional diffusion-wave equation. In [5], the authors used the Sinc-Legendre collocation method to a numerical solution for a class of fractional convection-diffusion equation.

The theory of nonlinear dispersive wave motion has recently undergone much study, especially by Whitham [6]. It can be shown that the theory of water waves for the case of shallow water and waves of small amplitude can be approximately described by the Korteweg-de Vries equation 


$$
\frac{\partial u(x, t)}{\partial t}+(c+u(x, t)) \frac{\partial u(x, t)}{\partial x}+\beta \frac{\partial^{3} u(x, t)}{\partial x^{3}}=0, \quad(x, t) \in \mathbb{R} \times(0, T)
$$

where $c$ and $\beta$ are given constants, and $u$ gives the height of a wave above some equilibrium level. Since the amplitude of these waves is assumed to be small, it can serve as a perturbation parameter. These problems have been studied by many authors [7-9]. However, they used a formal perturbation technique. Sometimes called multiscale expansion, or, using evens functions techniques, as in [10]. One aspect that has been investigated is the linearized form of Equation (1):

$$
\frac{\partial u(x, t)}{\partial t}+c \frac{\partial u(x, t)}{\partial x}+\beta \frac{\partial^{3} u(x, t)}{\partial x^{3}}=0, \quad(x, t) \in \mathbb{R} \times(0, T)
$$

which has traveling wave solutions $u(x, t)=a \cos (k x-\omega t)$, where $a$ is constant and $\omega=\omega(k)=c k-\beta k 3$. The existence of traveling wave solutions to (2) already has been studied in [11]. If we drop the third derivative term in (1), we have

$$
\frac{\partial u(x, t)}{\partial t}+(c+u(x, t)) \frac{\partial u(x, t)}{\partial x}+\beta \frac{\partial^{3} u(x, t)}{\partial x^{3}}=0, \quad(x, t) \in \mathbb{R} \times(0, T)
$$

which is a quasi-linear first-order wave equation whose wave speed depends on the amplitude and has the implicit solutions $u(x, t)=a \cos [k x-k(c+u) t]$. If $c=0 ; \beta=1$ in Equation $\mathrm{k}(1)$ we get another form of Korteweg-de Vries equation

$$
\frac{\partial u(x, t)}{\partial t}+u(x, t) \frac{\partial u(x, t)}{\partial x}+\beta \frac{\partial^{3} u(x, t)}{\partial x^{3}}=0,
$$

This nonlinear equation admits traveling wave solutions of different types. One particular type of traveling wave that arises from the Korteweg-de Vries equation is the soliton, or solitary wave. The same equation (3) has also come up in the theory of plasma and several other branches of physics.

In recent years, there has been a growing interest in the field of fractional calculus. Oldham and Spanier [1], Miller and Ross [2], and Podlubny [3] provide the history and a comprehensive treatment of this subject. Fractional calculus is the field of mathematical analysis, which deals with the investigation and applications of integrals and derivatives of arbitrary order, which can be real or complex. Many applications of fractional calculus can be found in turbulence and fluid dynamics, stochastic dynamical systems, plasma physics and controlled thermonuclear fusion, nonlinear control theory, image processing, nonlinear biological systems, for more see [12] and the references therein. Indeed, it provides several potentially useful tools for solving differential equations. It is important to solve time fractional partial differential equations. It was found that fractional time derivatives arise generally as infinitesimal generators of the time evolution when taking along time scaling limit. Hence, the importance of investigating fractional equations arises from the necessity to sharpen the concepts of equilibrium, stability states, and time evolution in the long time limit. In general, there exists no method that yields an exact solution for nonlinear fractional partial differential equations. There has been some attempt to solve linear problems with multiple fractional derivatives. In the present paper, we consider the fractional Korteweg-de Vries Equation

$$
\frac{\partial^{\alpha} u(x, t)}{\partial t^{\alpha}}+(c+u(x, t)) \frac{\partial u(x, t)}{\partial x}+\beta \frac{\partial^{3} u(x, t)}{\partial x^{3}}=0, \quad(x, t) \in \mathbb{R} \times(0, T)
$$

with the initial condition

$$
u(x, 0)=f(x), \quad x \in \mathbb{R}
$$

Following [13], we construct the solution using different approach, that is based on using collocation techniques. The method combining a finite difference approach in the time-fractional direction, and the Sinc-Collocation in the space direction, where the derivatives are replaced by the necessary matrices, and a system of algebraic equations is obtained to approximate solution of the problem.

Many researchers have used various numerical methods to solve Korteweg-de Vries Equation. Al-Khaled [14], uses Sinc-Galerkin method to find a numerical solution of the Korteweg-de Vries Equation. The method results in an iterative scheme of an error of order $\mathcal{O}(\exp (-c / h))$ for some positive constants $c, h$. 
In [15], the $\mathrm{KdV}$ equation is transformed into an equivalent integral equation, and a Sinc-collocation procedure is developed for the integral equation.

In this paper, following the same idea as in [13], we will use Sinc-methodology to study the solution of equation (4). We will present a simple numerical method that uses finite differences to replace the first-order time derivative with a fractional derivative of $\operatorname{order} \alpha$, with $0<\alpha \leq 1$. The physical interpretation of the fractional derivative is that it represents a degree of memory in the diffusing material. The Sinc-collocation method will be used in the space direction. The main idea is to replace differential and integral equations by their Sinc approximations. The ease of implementation coupled with the exponential convergence rate have demonstrated by viability of this method.

To enable us to follow the solution of the Fractional Burgers' equation [17, 20, 23], many definitions and studies of fractional calculus have been proposed in the last two centuries. These definitions include, Riemman-Liouville, Weyl, Reize, Campos, Caputa, and Nishimoto fractional operator. The Riemann-Liouville definition of fractional derivative operator $J_{a}^{\alpha}$ which is defined in [1]. The Riemann-Liouville derivative has certain disadvantages when trying to model real-world phenomena with fractional differential equations. Therefore, we shall introduce a modified fractional differentiation operator $D^{\alpha}$ proposed by Caputo's (see, [2]). Sinc function that will be used in this project, are discussed in Stenger [15] and by Lund [24].

\section{CONSTRUCTION OF THE SCHEME}

In this section, finite difference method scheme and Sinc-Collocation method is used for solving the Fractional Burgers' equation (4). In the analysis of the numerical method that follows, we will assume that problem (4)-(5) has a unique and sufficiently smooth solution.

\subsection{Fractional time-derivative}

This sub-section is devoted to a description of the operational properties of the purpose of acquainting with sufficient fractional calculus theory. Many definitions and studies of fractional calculus have been proposed in the last two centuries. These definitions include, Riemman-Liouville, Weyl, Reize, Campos, Caputa, and Nishimoto fractional operator. As mentioned in [19], the Riemann-Liouville derivative has certain disadvantages when trying to model real-world phenomena with fractional differential equations. Therefore, we shall introduce now a modified fractional differentiation operator $D^{\alpha}$ proposed by Caputo in his work on the theory of viscoelasticity [22]. The Caputo fractional derivative is considered in the Caputo sense. For more details on the geometric and physical interpretation for fractional derivatives of both Riemann-Liouville and Caputo types see [22].

Definition 1 For $m$ to be the smallest integer that exceeds $\alpha$, the Caputo fractional derivatives of order $\alpha>0$ is defined as

$$
D^{\alpha} u(x, t)=\frac{\partial^{\alpha} u(x, t)}{\partial t^{\alpha}}=\left\{\begin{array}{l}
\frac{1}{\Gamma(m-\alpha)} \int_{0}^{t}(t-\tau)^{m-\alpha-1} \frac{\partial^{m} u(x, \tau)}{\partial \tau^{m}} d \tau, \quad m-1<\alpha<m \\
\frac{\partial^{m} u(x, t)}{\partial t^{m}}, \quad \alpha=m \in N
\end{array}\right.
$$

For mathematical properties of fractional derivatives and integrals one can consult the above mentioned references.

\subsection{Discretization the time-fractional derivative}

Consider the one-dimensional time-fractional Burgers' equation (4). Following [16], we introduce a finite difference approximation to discretize the time-fractional derivative. Let $t_{k}=k \Delta t, k=0,1, \ldots K$, where $\Delta t=T / K$ is the time step. Now by using the definition of Caputo time-fractional derivative, and since $0<\alpha \leq 1$, in Definition 1 , we take $m=1$, then for $k=0,1, \ldots, K$, we have 


$$
\begin{aligned}
\frac{\partial^{\alpha} u\left(x, t_{k+1}\right)}{\partial t^{\alpha}} & =\frac{1}{\Gamma(1-\alpha)} \int_{0}^{t_{k+1}} \frac{\partial u(x, s)}{\partial s} \frac{d s}{\left(t_{k+1}-s\right)^{\alpha}}=\frac{1}{\Gamma(1-\alpha)} \sum_{j=0}^{k} \int_{t_{j}}^{t_{j+1}} \frac{\partial u(x, s)}{\partial s} \frac{d s}{\left(t_{k+1}-s\right)^{\alpha}} \\
& =\frac{1}{\Gamma(1-\alpha)} \sum_{j=0}^{k} \frac{u\left(x, t_{j+1}\right)-u\left(x, t_{j}\right)}{\Delta t} \int_{t_{j}}^{t_{j+1}} \frac{d s}{\left(t_{k+1}-s\right)^{\alpha}}+r_{\Delta t}^{k+1}
\end{aligned}
$$

where $r_{\Delta t}^{k+1}$ is the truncation error, that takes the form

$$
r_{\Delta t}^{k+1} \leq c_{u} \frac{1}{\Gamma(1-\alpha)} \sum_{j=0}^{k} \int_{t_{j}}^{t_{j+1}} \frac{\left(t_{j+1}-t_{j}-2 s\right)}{\left(t_{k+1}-s\right)^{\alpha}} d s+\mathcal{O}\left(\Delta t^{2}\right)
$$

It has been proved in [16] that $r_{\Delta t}^{k+1} \leq c_{u} \Delta t^{2-\alpha}$, where $c_{u}$ is a constant depending only on $u$. Set $\xi=t_{j+1}-s$, and since $t_{k}=k \Delta t, k=0,1, \ldots, K$, we have: As $s=t_{k}$, then $\xi=t_{k+1}-t_{k}=\Delta t(k-j)=t_{k-j}$, and as $s=t_{j+1}$, then $\xi=t_{k-j}$. Therefore,

$$
\begin{aligned}
\frac{\partial^{\alpha} u\left(x, t_{k+1}\right)}{\partial t^{\alpha}} & \approx \frac{1}{\Gamma(1-\alpha)} \sum_{j=0}^{k} \frac{u\left(x, t_{j+1}\right)-u\left(x, t_{j}\right)}{\Delta t} \int_{t_{k-j}}^{t_{k-j+1}} \frac{d \xi}{\xi^{\alpha}}=\frac{1}{\Gamma(1-\alpha)} \sum_{j=0}^{k} \frac{u\left(x, t_{k-j+1}\right)-u\left(x, t_{k-j}\right)}{\Delta t} \int_{t_{j}}^{t_{j+1}} \frac{d \xi}{\xi^{\alpha}} \\
& =\frac{1}{\Gamma(2-\alpha)} \sum_{j=0}^{k} \frac{u\left(x, t_{k-j+1}\right)-u\left(x, t_{k-j}\right)}{\Delta t}\left[\left(t_{j+1}\right)^{1-\alpha}-\left(t_{j}\right)^{1-\alpha}\right] \\
& =\frac{1}{\Gamma(2-\alpha)} \sum_{j=0}^{k} \frac{u\left(x, t_{k-j+1}\right)-u\left(x, t_{k-j}\right)}{\Delta t}\left[(\Delta t)^{1-\alpha}\left[(j+1)^{1-\alpha}-(j)^{1-\alpha}\right]\right]
\end{aligned}
$$

To simplify the above result, we introduce the notations $b_{j}=(j+1)^{1-\alpha}-j^{1-\alpha}, j=0,1,2, \ldots, K$, and we define the discrete fractional differential operator

$$
\frac{\partial^{\alpha} u\left(x, t_{k+1}\right)}{\partial t^{\alpha}} \approx \frac{1}{(\Delta t)^{\alpha} \Gamma(2-\alpha)} \sum_{j=0}^{k} b_{j}\left[u\left(x, t_{k-j+1}\right)-u\left(x, t_{k-j}\right)\right]
$$

In equation (4), replace $t$ by $t_{k+1}$, and plug in into equation (6), we obtain the approximation

$\frac{1}{\Gamma(2-\alpha)(\Delta t)^{\alpha}} \sum_{j=0}^{k} b_{j}\left[u\left(x, t_{k-j+1}\right)-u\left(x, t_{k-j}\right)\right]=-\beta \frac{\partial^{3} u\left(x, t_{k+1}\right)}{\partial x^{3}}-\left(c+u\left(x, t_{k+1}\right)\right) \frac{\partial u\left(x, t_{k+1}\right)}{\partial x}, k=0,1, \ldots, K$,

or,

$$
\sum_{j=0}^{k} b_{k-j}\left[u\left(x, t_{j+1}\right)-u\left(x, t_{j}\right)\right]=(\Delta t)^{\alpha} \Gamma(2-\alpha)\left(-\beta \frac{\partial^{3} u\left(x, t_{k+1}\right)}{\partial x^{3}}-\left(c+u\left(x, t_{k+1}\right)\right) \frac{\partial u\left(x, t_{k+1}\right)}{\partial x}\right)
$$

Let $u_{k}(x)$ be an approximation to $u\left(x, t_{k}\right)$, and call $\nu=\Gamma(2-\alpha)(\Delta t)^{\alpha}$, then for $k=0,1, \ldots, K$, the above equation becomes

$$
\sum_{j=0}^{k} b_{k-j} u_{j+1}(x)=\sum_{j=0}^{k} b_{k-j} u_{j}(x)+\nu\left(-\beta \frac{d^{3}}{d x^{3}} u_{k+1}(x)-\left(c+u_{k+1}(x)\right) \frac{d}{d x} u_{k+1}(x)\right)
$$




\subsection{Sinc-Collocation}

The goal of this sub-section is to recall notations and definitions of the Sinc function that will be used in this paper. These are discussed in $[15,24,21]$. The sinc function is defined on the whole real line, by

$$
\operatorname{sinc}(z) \equiv \begin{cases}\frac{\sin (\pi z)}{\pi z}, & z \neq 0 \\ 1, & z=0\end{cases}
$$

For $h>0$ and $k=0, \pm 1, \pm 2, \ldots$, the translated sinc function with evenly spaces nodes are given by

$$
S(k, h)(z) \equiv \begin{cases}\frac{\sin \left[\left(\frac{\pi}{h}\right)(z-k h)\right]}{\left[\left(\frac{\pi}{h}\right)(z-k h)\right]}, & z \neq k h, \\ 1, & z=k h .\end{cases}
$$

Definition 2 Let $d>0$, and let $\mathcal{D}_{d}$ denote the region $\{z=x+i y:|y|<d\}$ in the complex plane $\mathbb{C}$, and $\phi$ the conformal map of a simply connected domain $\mathcal{D}$ in the complex domain onto $\mathcal{D}_{d}$ such that $\phi(a)=-\infty$ and $\phi(b)=\infty$, where $a$ and $b$ are boundary points of $\mathcal{D}$, i.e., $a, b \in \partial \mathcal{D}$. Let $\psi$ denote the inverse map of $\phi$, and let the arc $\Gamma$, with endpoints $a$ and $b(a, b \notin \Gamma)$, given by $\Gamma=\psi(-\infty, \infty)$. For $h>0$, let the points $x_{k}$ in $\Gamma$ be given by $x_{k}=\psi(k h), k \in \mathbb{Z}, \rho(z)=\exp (\phi(z))$.

The sinc-collocation procedure for equation (8), begins by selecting composite sinc functions, appropriate to the interval $(a, b)$, as the basis function for the expansion of the approximate solution for $u(x)$. For the present paper the interval $\Gamma$ in the above definition is $(-\infty, \infty)$. Therefore, to approximate the first and third derivative we take $\phi(x)=x$. The basis functions are derived from the composite translated sinc functions

$$
S_{i}(x)=S(i, h) \circ \phi(x)=\operatorname{sinc}[(\phi(x)-i h) / h]
$$

$S_{i}(x)=S(i, h) \circ \phi(x)=S(i, h)(x)$ in equation (11) define the basis element for equation (8) on the interval $(-\infty, \infty)$. Here $h$ is the mesh size, the sinc grid points $x_{n} \in(-\infty, \infty)$ will be denoted by $x_{n}$ because they are real. The inverse images of the equispaced grids are $x_{n}=\phi^{-1}(n h)$. Also for positive integer $N$, define

$$
C_{N}(f, h)(x)=\sum_{i=-N}^{N} f(i h) S(i, h) \circ \phi(x)=\sum_{i=-N}^{N} f(i h) S(i, h)(x) .
$$

To approximate the derivatives of a function $f(x)$ by the sinc expansion, the derivatives of sinc functions be evaluated at the nodes will be needed $[15,24]$. In particular, the following convenient notation will be useful in formulating the discrete system.

$$
\begin{aligned}
& \delta_{k-j}^{(0)}=\left\{\begin{array}{l}
1, j=k \\
0, j \neq k,
\end{array} \delta_{k-j}^{(1)}=h \frac{d}{d x}\left[\left.S(j, h) \circ \phi(x)\right|_{x=x_{k}}=\left\{\begin{array}{l}
0, j=k \\
\frac{(-1)^{j-k}}{j-k}, j \neq k
\end{array}\right. \text { and, }\right.\right. \\
& \delta_{k-j}^{(3)}=h^{3} \frac{d^{3}}{d x^{3}}\left[\left.S(j, h) \circ \phi(x)\right|_{x=x_{k}}=\left\{\begin{array}{l}
0, j=k \\
\frac{(-1)^{j-k}}{(j-k)^{3}}\left[6-\pi^{2}(j-k)^{2}\right], j \neq k
\end{array}\right.\right.
\end{aligned}
$$

Now, we expand $u_{k}(x), k=0,1, \ldots, K-1$ by Sinc function

$$
u_{k}(x)=\sum_{i=0}^{N} C_{i}^{k} S_{i}(x), k=1, \ldots, K
$$

where $C_{0}^{k}, C_{1}^{k}, \ldots, C_{N}^{k}$ are unknown coefficients to be determined. Substitute equation (13) into equation (8), for $k=0,1, \ldots, K-1$, we have

$\sum_{j=0}^{k} \sum_{i=0}^{N} C_{i}^{j+1} S_{i}(x) b_{k-j}=\sum_{j=0}^{k} \sum_{i=0}^{N} C_{i}^{j} S_{i}(x) b_{k-j}-\nu \beta \sum_{i=0}^{N} C_{i}^{k+1} S_{i}^{\prime \prime \prime}(x)-\nu\left(c+\sum_{i=0}^{N} C_{i}^{k+1} S_{i}(x)\right) \sum_{i=0}^{N} C_{i}^{k+1} S_{i}^{\prime}(x)$. 
To find the unknown coefficients $C_{0}^{k}, C_{1}^{k}, \ldots, C_{N}^{k}$, Sinc collocation method with collocation points $x_{n}$ is applied to equation (14), and for $k=1, \ldots, K$ yield

$$
\begin{array}{r}
\sum_{j=0}^{k} \sum_{i=0}^{N} C_{i}^{j+1} S_{i}\left(x_{n}\right) b_{k-j}=\sum_{j=0}^{k} \sum_{i=0}^{N} C_{i}^{j} S_{i}\left(x_{n}\right) b_{k-j}-\nu \beta \sum_{i=0}^{N} C_{i}^{k+1} S_{i}^{\prime \prime \prime}\left(x_{n}\right) \\
-\nu\left(c+\sum_{i=0}^{N} C_{i}^{k+1} S_{i}\left(x_{n}\right)\right) \sum_{i=0}^{N} C_{i}^{k+1} S_{i}^{\prime}\left(x_{n}\right) .
\end{array}
$$

where $u_{0}(x)$ can be obtained from the initial condition as follows:

$$
u_{0}(x)=u\left(x, t_{0}\right)=f(x) .
$$

Equations (15)-(16) generate a set of $N+1$ algebraic equations which can be solved to find the unknown coefficients $C_{0}^{k}, C_{1}^{k}, \ldots, C_{N}^{k}$.

\subsection{Matrix Form of the proposed method}

In order to find the matrix form of the proposed method, define Toeplitz matrices $I_{j k}^{(q)}, q=0,1,3$ whose $j k$ th entry is given by $\delta_{k-j}^{(q)}$. Note that the matrices $I_{j k}^{(1)}, I_{j k}^{(3)}$ are skew symmetric, and the matrix $I_{j k}^{(0)}$ is an identity matrix. By separating the $\mathrm{k} t h$ term from the first term of left hand side of equation (15), and for $n=0,1, \ldots, N-1$, we obtain

$$
\begin{aligned}
\sum_{i=0}^{N} C_{i}^{k+1} S_{i}\left(x_{n}\right) b_{0} & +\nu \beta \sum_{i=0}^{N} C_{i}^{k+1} S_{i}^{\prime \prime \prime}\left(x_{n}\right)=\sum_{j=0}^{k} \sum_{i=0}^{N} C_{i}^{j} S_{i}\left(x_{n}\right) b_{k-j}-\sum_{j=0}^{k-1} \sum_{i=0}^{N} C_{i}^{j+1} S_{i}\left(x_{n}\right) b_{k-j} \\
& +\nu\left(c+\sum_{i=0}^{N} C_{i}^{k+1} S_{i}\left(x_{n}\right)\right) \sum_{i=0}^{N} C_{i}^{k+1} S_{i}^{\prime}\left(x_{n}\right) .
\end{aligned}
$$

In the above equation, collect terms, and making the same upper indices, we have

$$
\begin{aligned}
\sum_{i=0}^{N} C_{i}^{k+1}\left[S_{i}\left(x_{n}\right) b_{0}-\nu \beta S_{i}^{\prime \prime \prime}\left(x_{n}\right)\right] & =\sum_{j=0}^{k} \sum_{i=0}^{N} C_{i}^{j} S_{i}\left(x_{n}\right)\left[b_{k-j}-b_{k+1-j}\right] \\
& +\nu\left(c+\sum_{i=0}^{N} C_{i}^{k+1} S_{i}\left(x_{n}\right)\right) \sum_{i=0}^{N} C_{i}^{k+1} S_{i}^{\prime}\left(x_{n}\right) .
\end{aligned}
$$

The matrix form can be obtained for $k=0,1, \ldots, K-1$ and $n=1,2, \ldots, N-1$ as

$$
M[C]^{k+1}=\sum_{\ell=0}^{k}\left(b_{k-\ell}-b_{k-\ell+1}\right) A_{d}[C]^{\ell} .
$$

where,

$$
\begin{aligned}
& M=\left[A_{d}-\nu \beta B-\nu A\left([C]^{k+1}\right)^{T} \circ\left(c-[C]^{k+1}\right)\right] \\
& {[C]^{k}=\left[C_{0}^{k}, C_{1}^{k}, \ldots, C_{N}^{k}\right]^{T}, \text { here } T \text { is the transpose. }} \\
& A_{d}=\left[I_{i j}^{(0)}: i=2, \ldots, N-1, j=1, \ldots, N, \text { and } 0 \text { elsewhere }\right]_{N \times N}, \\
& A=\left[I_{i k}^{(1)}: i=2, \ldots, N-1, j=1, \ldots, N, \text { and } 0 \text { elsewhere }\right]_{N \times N}, \text { and } \\
& B=\left[I_{i k}^{(3)}: i=2, \ldots, N-1, j=1, \ldots, N, \text { and } 0 \text { elsewhere }\right]_{N \times N} .
\end{aligned}
$$


The symbol $\circ$ in matrix $M$ means the componentwise multiplication. We can obtain the coefficients $C_{i}^{k}, i=0,1, \ldots, N$ of the approximate solution by solving the linear system using an iterative technique. The convergence of the of the series $u^{k}$ to $u$ with increasing the number of collocation points $N$, we require two necessary conditions, first, the function $u$ must belong to the Paley Wiener space [15], and second, $u$ must defined on the whole real line. For the analysis of the stability for the Sinc method for solving the fractional Burgers' equation, we may refer readers to resemble similar proof in [13].

\section{NUMERICAL RESULTS}

Here, we obtain some numerical results for the solutions of the fractional $\mathrm{KdV}$ equation (2). We use the parameters, $d=\pi / 2, N=16$ to check the performance for the solution of the fractional KdV equation. The computations associated with the example were performed using Mathematica.

Example 1 Consider the non-linear fractional KdV equation

$$
\frac{\partial^{\alpha} u(x, t)}{\partial t^{\alpha}}+6 u(x, t) \frac{\partial u(x, t)}{\partial x}+\frac{\partial^{3} u(x, t)}{\partial x^{3}}=0, \quad 0 \leq x \leq 1,0<\alpha \leq 1, t>0
$$

It is be noted that the exact solution for any $\alpha$ has the closed form [18]

$$
u(x, t ; \alpha)=\operatorname{sech}^{2}\left(\frac{1}{\sqrt{2}}\left[x-\frac{2 t^{\alpha}}{\Gamma[1+\alpha]}\right]\right)
$$

In order to illustrate the approximate solution is efficiency and accurate. Some numerical values for given explicit values of the parameters $t$ and $\alpha$ for fixed $x=0.2$ are depicted in Table 1 . From the numerical values in Table 1 , it can be seen that the exact solution $(\alpha=1)$ is quite close to the approximate solution when $\alpha=0.95$. Also, in Figure 1 it is observed that the values of the approximate solution at different $\alpha$ 's has the same behavior as those obtained using equation (20) for which $\alpha=1$. This shows the approximate solution is efficiency. In the theory of fractional calculus, it is obvious that when the fractional derivative $\alpha(m-1<\alpha \leq m)$ tends to positive integer $m$, then the approximate solution continuously tends to the exact solution of the problem with derivative $m=1$. A closer look at the values in Tables 1 and 2 , we observe that our approach do have this characteristic. Figures 2 and 3 shows the approximate solution for $\alpha=0.25$ and $\alpha=1$ respectively. Comparison of Figures 2 and 3 shows that the solution continuously depends on the fractional derivatives.

Table 1. Numerical Results obtained by equation (20) for various values of $\alpha$ when $x=2$

\begin{tabular}{ccccccc}
\hline$t$ & $\alpha=1$ & $\alpha=0.95$ & $\alpha=0.75$ & $\alpha=0.50$ & $\alpha=0.25$ & $\alpha=0.10$ \\
\hline 0.1 & 0.269740 & 0.279333 & 0.336412 & 0.480254 & 0.759333 & 0.947435 \\
0.2 & 0.341487 & 0.358411 & 0.450034 & 0.634307 & 0.874187 & 0.978219 \\
0.3 & 0.426435 & 0.449797 & 0.566152 & 0.756832 & 0.935571 & 0.990783 \\
0.4 & 0.523486 & 0.551760 & 0.680689 & 0.852402 & 0.970527 & 0.996662 \\
0.5 & 0.629290 & 0.666666 & 0.786983 & 0.922553 & 0.989626 & 0.999259 \\
\hline
\end{tabular}

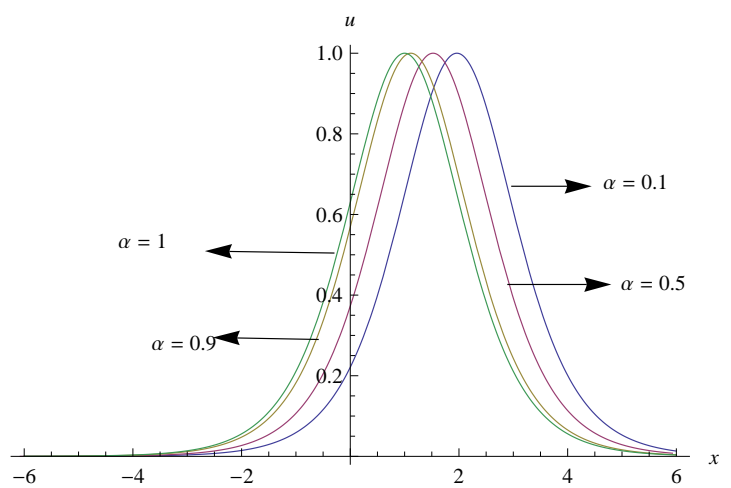

Figure 1. The approximate solution when $t=0.2$, for different values of $\alpha$ 


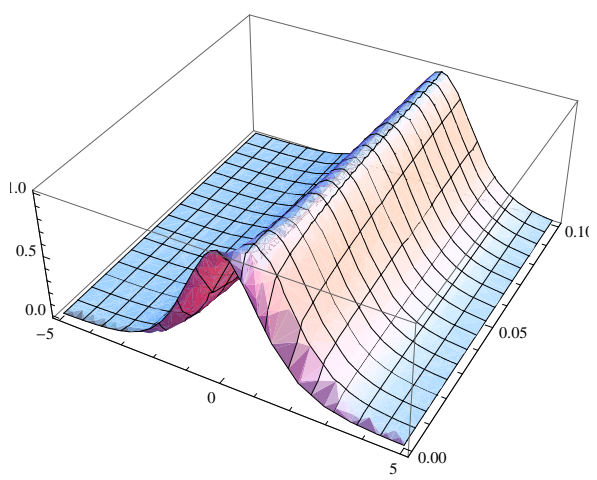

Figure 2. The approximate solution when $\alpha=0.25$

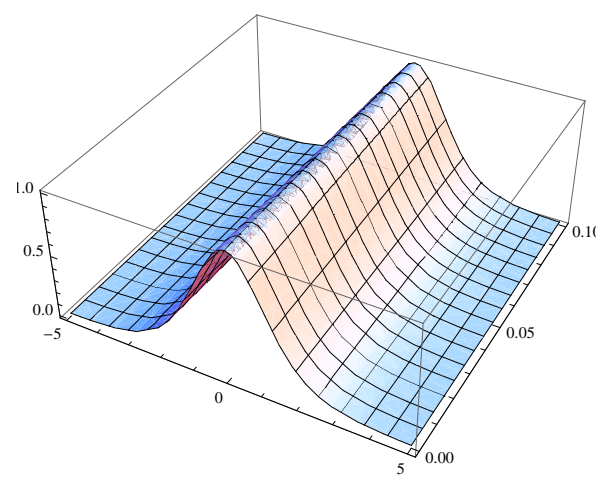

Figure 3. The approximate solution when $\alpha=1.0$

\section{DISCUSSION AND CONCLUSIONS}

The Sinc-Collocation method appears to be very promising for solving the fractional Burgers' equation. An important advantage to be gained from the use of this method is the ability to produce very accurate results. The example presented demonstrate the accuracy of the method, which is an improvement over current methods such as finite elements and finite difference methods. This feature show the method to be an attractive for numerical solutions to the fractional Burgers' equation. We conclude, with confidence, that the collocation using Sinc basis can be considered as a beneficial method for solving a broad class of fractional nonlinear partial differential equations. The study of these equations will be the matter of furthers investigations.

\section{REFERENCES}

[1] K. B. Oldham, J. Spanier, "The Fractional Calculus," Academic Press, New York, 1974.

[2] K. S. Miller, B. Ross, "An introduction to the Fractional Calculus and Fractional Differential equations," John Wiley and Sons Inc. New York, 1993.

[3] I. Podlubny, "Fractional Differential Equations," Academic Press, New York, 1999.

[4] Kamel Al-Khaled, Shaher Momani, "An approximate solution for a fractional diffusion-wave equation using the decomposition method," J. Comput. Appl. Math, vol. 165, pp. 473-483, 2005.

[5] A. Saadamandi, M. Dehghan, M.-Reza Aziz, "The Sinc-Legendre collocation method for a class of fractional convection-diffusion equations with variable coefficients," Commun Nonlinear Sci. Numer. Simulat. vol. 17, pp. 4125-4136, 2012.

[6] G.B. Whitham, "Linear and Nonlinear Waves," Wiley-Interscience, New York, 1974.

[7] R. Grimshaw, H. Mitsudera, "Slowly varying solitary wave solutions of the perturbed Korteweg-de Vries equation revisited," Stud. Appl. Math. vol. 90, pp. 75-86, 1993.

[8] Kamel Al-Khaled, "Numerical study of Fisher's reaction-diffusion equation by the Sinc collocation method," J. Comput. Appl. Math., vol. 137, pp. 245-255, 2001.

[9] Y. Kodama, M. Ablowitz, "Perturbations of solitons and solitary waves," Stud. Appl. Math., vol. 64, pp. 225-245, 1994.

[10] T. Ogawa, H. Suzuki, "On the spectra of pulses in a nearly integrable system," SIAM J. Appl. Math. vol. 57 (2), pp. 485-500, 1997.

[11] N.M. Ercolani, D.W. Mclaughlin, H. Roitner, "Attractors and transients for a perturbed Kdv equation," $A$ nonlinear spectral analysis, J. Nonlinear Sci., vol. 3, pp. 477-539, 1993.

[12] Yanqin Liu, Zhaolli Li, Yueyun Zhang, "Homotopoy Perturbation method for fractional biological population equation," Fractional Differential Calculus, vol. 1, No. 1, pp. 117-124, 2011.

[13] Marwan Alquran, Kamel Al-Khaled, Tridip Sardar, Joydev Chattopadhyay, "Revisited Fisher's Equation in a new outlook: A fractional derivative approach," Physica A: Statistical Mechanics and its Applications, 438, pp. 81-93, 2015.

[14] Kamel Al-Khaled, "Sinc numerical solution for solitons and solitary waves," J. Comput. Appl. Math., 130, pp. 283-292, 2001. 
[15] F. Stenger, "Numerical Methods Based on Sinc and Analytic Functions," Springer-Verlag, New York, 1993.

[16] Yumin Lin, Chuanju Xu, "Finite difference/special approximations for the time-fractional diffusion equation," J. Comput. Phys., 225, pp. 1533-1552, 2007.

[17] J, M., Burgers', "Application of a model system to illusrate some points of the statistical theory of free turbulance," Proceedings of the Royal Academy of Sciences of Amsterdam, vol. 43, pp. 2-12,1940.

[18] Najeeb Alam Khan, Asmat Ara, "Numerical solutions of time-fractional Burgers equations, a comparison between generalized differential transform technique and homotopy perturbation method," Inter. J. of Numer. Methods for Heat and Fluid Flow, Vol. 22, No. 2, pp. 175-193, 2012.

[19] I. Podlubny, "Gemmetric and physical interpretaion of fractional integration and fractional differentaition," Frac. Calc. Appl. Anal., vol. 5, pp. 367-386, 2002.

[20] Yufeng Xu, Om P. Agrawal, "Numerical solutions and analysis of diffusion for new generalized fractional Burgers equation," Fractional Calculus and Applied Analysis, vol. 16, No. 3, pp. 709-736, 2013.

[21] Baumann Gerd, Stenger Frank, "Fractional calculus and Sinc methods," Frac. Calc. Appl. Anal., vol. 14, No. 4, pp. 568-622, 2011.

[22] M. Caputo, "Linear models of dissipation whose $Q$ is almost frequency independent-II," Geophys. J. R. Astron. Soc., vol. 13, pp. 529-539, 1967.

[23] Amar G., Noureddine D., "Existence and uniqueness of solution to fractional Burgers' equation," Acta Univ. Apulensis, vol. 21, pp. 161-170, 2010.

[24] J. Lund and Bowers K. L, "Sinc methods for quadrature and differential equations," SIAM, Philadelphia, 1992.

\section{BIOGRAPHY OF AUTHOR}

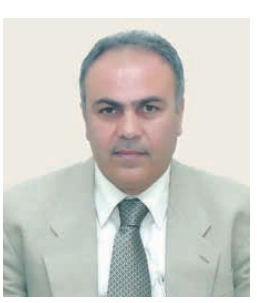

Kamel Al-Khaled Full Professor, Department of Mathematics and Statistics, Faculty of Science and Arts, Jordan University of Science and Technology, Irbid 22110, Jordan. email:kamel@just.edu.jo 